\title{
Scoping Review of Off-Label Topical Analgesia in Palliative, Hospice and Cancer Care: Towards Flexibility in Evidence-Based Medicine
}

\author{
Baraa $O$ Tayeb (D) ${ }^{1,2}$ \\ Jennifer A Winegarden $\left(\mathbb{D}^{3}\right.$ \\ Rawabi A Alashari ${ }^{4,5}$ \\ Moudi Alasmari $\mathbb{D}^{6,7}$ \\ Jonathan Winegarden ${ }^{8}$ \\ Faisal Boker ${ }^{9}$ \\ Abdulaziz Halawi ${ }^{2}$ \\ Amy Lapidow ${ }^{10}$ \\ Ylisabyth S Bradshaw" \\ Daniel B Carr (D) ${ }^{\prime \prime}$ \\ 'Department of Anesthesiology and \\ Critical Care, King Abdulaziz University \\ Faculty of Medicine, Jeddah, Saudi Arabia; \\ ${ }^{2}$ King Abdulaziz University Hospital, \\ Jeddah, Saudi Arabia; ${ }^{3}$ Department of \\ Medicine, The Medical Team Hospice, \\ Livonia, MI, USA; ${ }^{4}$ Department of \\ Pharmacology, King Abdulaziz University, \\ Faculty of Medicine, Jeddah, Saudi Arabia \\ ${ }^{5}$ Department of Pharmaceutical Science, \\ MCPHS University, Boston, MA, USA; \\ ${ }^{6}$ College of Medicine, King Saud bin \\ Abdulaziz University for Health Sciences, \\ Jeddah, Saudi Arabia; ${ }^{7}$ King Abdullah \\ International Medical Research Center \\ (KAIMRC), Jeddah, Saudi Arabia; \\ ${ }^{8}$ Department of Psychiatry and Psychology, \\ Mayo Clinic, Rochester, MN, USA; \\ ${ }^{9}$ Emergency Department, King Abdulaziz \\ Medical City-Ministry of National Guard \\ Health Affairs, Jeddah, Saudi Arabia; ${ }^{10}$ Tufts \\ Hirsh Health Sciences Library, Boston, MA, \\ USA; " Department of Public Health and \\ Community Medicine, Tufts University \\ School of Medicine, Boston, MA, USA
}

Correspondence: Baraa O Tayeb Faculty of Medicine, King Abdulaziz University, P.B. Box 80215 , Jeddah, 21589 Saudi Arabia

Tel +966-2-640-1000 (ext. I-I0199/10209)

$\mathrm{Fax}+966-2-640-8335$

Email btayeb@kau.edu.sa
Purpose: Scoping reviews address the nature of the literature per se rather than inferring evidence-based treatment guidelines. Scoping reviews of the published literature are intended to describe the aggregated nature of the evidence surrounding some agent or intervention, in contrast to systematic reviews that seek when possible to guide clinical practice. We conducted a scoping review to identify reports of potential clinical utility of off-label topical analgesics and adjuvants when FDA-approved treatments have proven inadequate.

Methods: We performed a comprehensive search of three databases (PubMed, Web of Science and Embase) for articles dating from 1947 to the present. Mindful that FDAapproved and WHO-recommended analgesic medications often prove inadequate for individual patients in extremis with palliative, hospice or cancer pain, we used broad, structured inclusion criteria to retrieve articles.

Results: We retrieved 12,100 articles; after screening, we had 39 reports addressing 19 different topical agents out of the 32 chemical entities. Our scoping review disclosed evidence about agents that might not have met inclusion criteria for clinical practice guidelines.

Discussion: Although generally considered lower quality evidence, case reports or series present suggestions for diverse topical medications to manage pain in challenging circumstances when high-quality evidence for agents and routes of administration is lacking.

Conclusion: Patients with the greatest need for evidence to identify and guide lesser-used agents during aggressive pain management are the most difficult to enroll and follow in standardized, controlled and/or blinded clinical trials. This scoping review identifies medications, dosages, and routes of topical agents reported to be effective in these often-challenging circumstances. Until larger and higher quality studies are completed, we must rely on the best available evidence even if of lower quality.

Keywords: topical medications, pain, analgesia, palliative care, hospice, cancer, evidencebased medicine, personalized medicine

\section{Introduction}

"Where there is pain, there the medicine goes" [Rumi, 13th century Sufi polymath and poet]

Uncontrolled pain is a significant problem in hospice and palliative medicine, and cancer care. ${ }^{1}$ A recent meta-analysis reported a pain prevalence in $38.9 \%$ of all patients with cancer and $66.4 \%$ of those with advanced, metastatic or terminal disease. $^{2}$ Often, standard therapies and guidelines prove inadequate and physicians turn to adjuvant pain medications, ${ }^{3}$ alternative routes of delivery, or interventional ${ }^{4}$ 
methods of pain control. Thus, the use of topical medications for acute, chronic and cancer-related pain has grown increasingly popular. Scoping reviews of the published literature are intended to describe the aggregated nature of the evidence surrounding some agent or intervention, in contrast to systematic reviews that seek when possible to guide clinical practice. $^{5}$ However, regulatory or quasigovernmental, eg, WHO, guidance concerning topical agents for hospice, palliative and cancer care pain regimens is hampered by a dearth of randomized and blinded controlled studies. ${ }^{6}$ A committee recently convened by the US National Academies of Science, Engineering and Medicine (NASEM) undertook a broad review of the use of topical medications for the treatment of pain. ${ }^{7}$ Their recommendations (published in 2020) spanned standard treatments in patients with acute (eg, musculoskeletal) or chronic (eg, neuropathic or inflammatory) noncancer pain. Also included in the NASEM review were patients whose neuropathic pain resulted from prior chemotherapy, but not from ongoing progression of underlying malignancies for which chemotherapy would be given. The patients assessed in the NASEM report included those studied in the context of clinical trials designed to minimize bias, or case reports if no higher quality evidence were available. The NASEM participants' desire to minimize methodological bias intentionally excluded multiple reports from consideration. Importantly, the focus of the NASEM report was upon patients or subjects whose skin was intact, thereby excluding patients whose pain arose from ulcerated cutaneous lesions, ie, who would be included in the present review.

Thus, we performed a comprehensive scoping review to focus upon patients receiving topical agents in the context of hospice, palliative or cancer-related care - few if any of whom would have been discussed in the NASEM report. Our goal was not to winnow down the articles retrieved during a comprehensive literature search. Instead, we wanted to be as inclusive as possible to call clinicians' attention to agents with which they might be less familiar and that are not FDA-approved for the treatment of cancer pain. Such topical agents might be considered for patients who had failed standard guidelines and who were running out of analgesic options as they approached life's end.

\section{Methods}

For the reasons described above, a "scoping review" (also called "mapping review" or "scoping study") seemed a more appropriate technique than the conventional systematic review to approach this still-growing, marginal literature. Scoping studies are "meant to 'map' relevant literature in a field of interest". 6,8 Arksey and O'Malley ${ }^{8}$ describe two "main differences between a systematic review and a scoping study":

- "A systematic review might typically focus on a well-defined question where appropriate study designs can be identified in advance, whilst a scoping study tends to address broader topics where many different study designs might be applicable."

- "The systematic review aims to provide answers to questions from a relatively narrow range of quality assessed studies, whilst a scoping study is less likely to seek to address very specific research questions nor consequently, to assess the quality of included studies."

To the above distinction, Peters et $\mathrm{al}^{9}$ add;

... scoping reviews are commonly used for 'reconnaissance' - to clarify working definitions and conceptual boundaries of a topic or field. Scoping reviews are therefore particularly useful when a body of literature has not yet been comprehensively reviewed, or exhibits a complex or heterogenous nature not amenable to a more precise systematic review of the evidence.

To accomplish our scoping study, we enlisted the support of a co-author (AL) with extensive experience as a research librarian supporting literature reviews. We went through six iterations of search terms before arriving at the final version (Appendix 1), based on the need to be comprehensive without generating an unmanageably large group of articles to screen and extract. We searched 32 active ingredients identified as chemical entities of interest for topical analgesia from the NASEM monograph (see below for search strategy). Following our initial review, we identified an additional five agents captured in the portion of our search that addressed pain control in palliative and hospice settings. These included menthol, sevoflurane, hyaluronic acid (Dermafill), hydrocolloid dressings and turmeric/curcumin. We included only English-language reports. We excluded articles that used iontophoresis, injection or any other methods of cutaneous drug administration other than having the medication applied onto the surface of the skin, wound or mucosa. In contrast to the NASEM methodology, we did not require that the skin be intact. We did not address agents' absorption through the skin 
nor their comparative effectiveness. Such analysis falls outside the framework of a scoping review, though the body of literature addressing mechanisms of action is increasing and may permit their future inclusion. ${ }^{10}$

Based upon our motive to assess novel or little-used agents, we excluded any articles about agents already United State Food and Drug Administration (USFDA)-approved to treat cancer-related pain and in common everyday use, eg, lidocaine-impregnated gauze, transdermal fentanyl or buprenorphine patches. We excluded articles that did not involve patients in hospice, palliative or cancer care or where it could not be discerned whether such patients were among the treated cases or groups. Grey literature was sought by employing broad inclusion criteria. Our search strategy resulted in identification of primary clinical data from case reports, case series, retrospective studies and prospective trials reported in journals, organizational newsletters, and program books of abstracts from scientific meetings. We excluded systematic or narrative reviews that lacked primary descriptions of palliative patients receiving non-US FDA approved medications .

We followed the PRISMA methodology ${ }^{11}$ (Figure 1) in which the successive phases of a systematic review are identification, screening, confirmation of eligibility, and inclusion. The initial search strategy (see Appendix 1) generated 12,100 "hits" after removal of duplicate entries from the three databases searched: PubMed, Embase and Web of Science. The titles and abstracts of each report were screened by two independent reviewers to confirm their relevance and suitability for more detailed screening. Each screened article was examined by two independent reviewers to confirm that it met primary inclusion/exclusion criteria. Data extracted from each of the screened
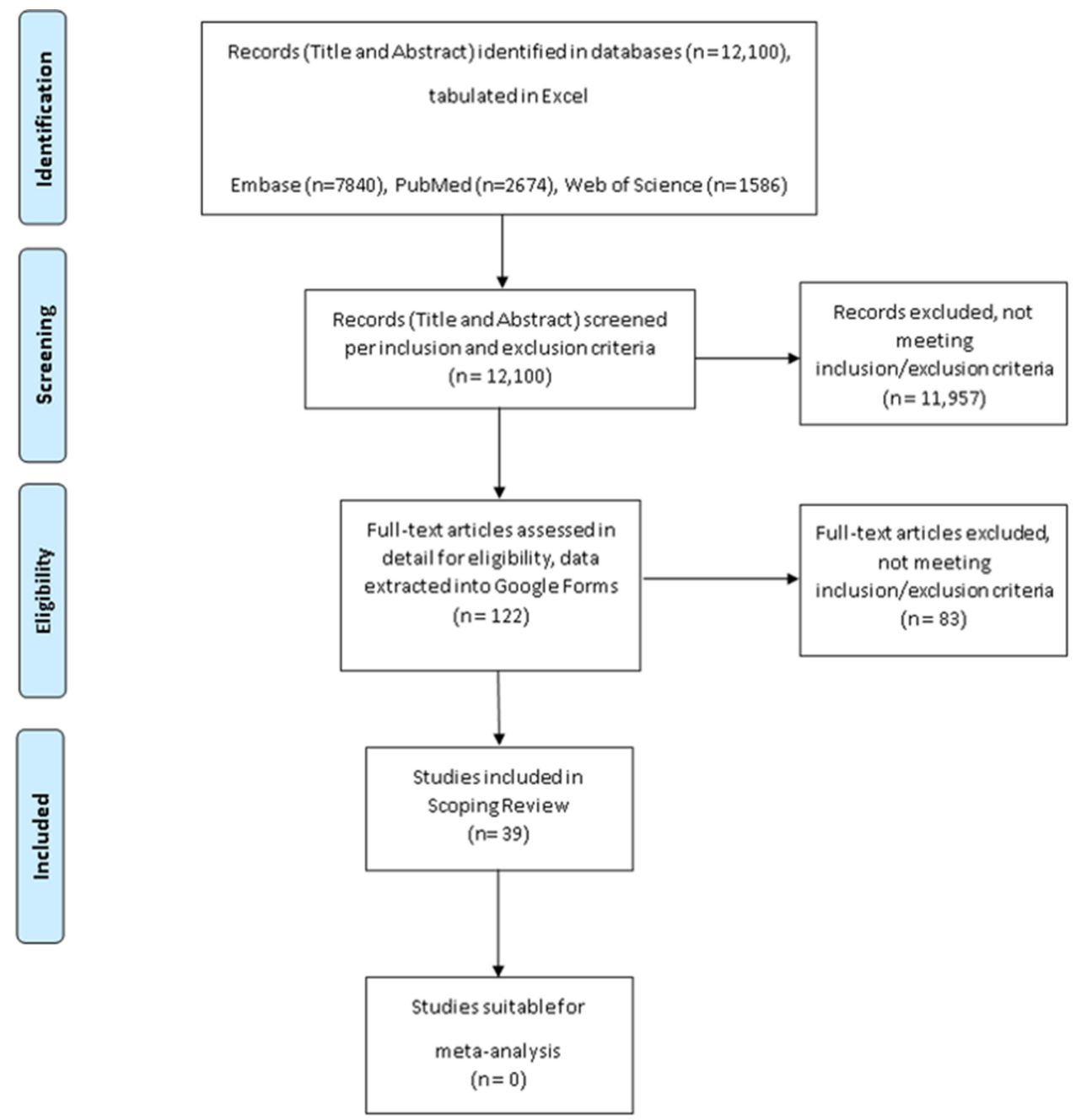

Figure I PRISMA 2009 Flow Diagram.

Notes: Adapted from Moher D, Liberati A, Tetzlaff J, Altman DG. Preferred reporting items for systematic reviews and meta-analyses: The PRISMA Statement. PLoS Med. 2009;6(7):el000097.' 
reports was tabulated in an Excel spreadsheet created for this purpose. A third reviewer was enlisted in case of disagreement between the two independent screening authors. If the third reviewer could not decisively state "include/exclude", the article was included in keeping with our motivation to include all studies whose results might benefit clinicians and patients in often dire circumstances.

The screening phase applied these inclusion criteria:

1. A primary or secondary end-point of the report was pain control.

2. The reported participants were (at least in part) receiving hospice or palliative care, and/or being treated for cancer-related pain. Our search terms included "hospice care," "hospice," "terminal care," "palliative therapy," "palliative nursing," "end of life," "terminal," and "palliative."

3. The active ingredient was one of the 32 agents examined in the NASEM report: amitriptyline, baclofen, benzhydrocodone, bupivacaine, cannabidiol, capsaicin, carbamazepine, clonidine, codeine, cyclobenzaprine, dexamethasone, doxepin, gabapentin, hydrocodone, hydromorphone, ketamine, levorphanol, meloxicam, memantine, meperidine, methadone, morphine, naltrexone, naproxen, nifedipine, orphenadrine, oxycodone, oxymorphone, pentoxifylline, tapentadol, topiramate, and tramadol. To these specific items, we added the search terms "analgesic agent," "analgesia," "pain," and "drug combination," to increase the sensitivity of our search.

4. The active agent was applied topically. Our search terms included "topical," "dermal," "skin," "transcutaneous," "rubs," "lotion," “gel," “patch," “cream," "transdermal," "transdermal drug administration," "percutaneous," "transmucosal," and "compound."

Exclusion criteria during this second phase of screening were:

1. The topical agent was already in customary, widespread daily use in dose regimens as approved for marketing by US or other nations' FDAs to treat cancer-related pain (fentanyl, lidocaine and buprenorphine).

2. The topical agent was applied using iontophoresis, phonophoresis or percutaneous injection.

3. The topical agent was administered intradermally or by percutaneous injection.

All articles identified during screening were retrieved in full except for three. The full texts of two of these were obtained through an international, interlibrary loan program; one of these ${ }^{12}$ was eligible for and included in subsequent data extraction. The article that could not be located in full was deemed ineligible on the basis of the information provided in its abstract. The data from all eligible articles was extracted using a Google Forms data sheet drafted for this study. This data included title, last name of first author, year of publication, type of study, endpoint(s), patient population, medication(s) used, medication formulation, application technique, medication concentration/dose/frequency, comparator medication/ placebo, initial participant number, final participant number, reasons for participant withdrawal, main results, and side effects. Once again, each article was assessed by two independent reviewers. The reports that were included are summarized in Appendix 2. It was obvious upon inspection that no quantitative syntheses, or pooling of results, was possible due to the heterogeneity in agents and diversity of the reports (including varied study designs and regimens tested). Across all agents, the durations of treatment were brief, reflecting clinical trial design and/or the limited life expectancy of many of the participants.

\section{Results}

Our initial literature search (Appendix 1) generated a total of 12,100 articles after being adjusted for duplications and incomplete citations. These articles were sourced from three databases: Embase (7840), PubMed (2674), and Web of Science (1586). A separate search for grey literature yielded no further studies, not surprising given how broad our initial search and inclusion criteria were. Each of the 12,100 articles was reviewed by two independent researchers who assessed their titles and abstracts. After this initial review, we applied our inclusion and exclusion criteria, resulting in 125 eligible articles. Two independent readers scrutinized each of these eligible articles, applied secondary exclusion and inclusion criteria, and extracted the data from the included articles onto a specially designed Google Forms extraction sheet. After confirming their eligibility, there remained a total of 39 reports $^{6,12-49}$ that were included (Appendix 1). These addressed 19 (Table 1) different topical agents out of the 32 chemical entities identified in the NASEM report as relevant to topical analgesia. The number of available studies and their generally weak nature are shown in Table 1. Appendix 2 provides specifics on the nature of the studies reported for individual agents according to their pharmacologic class. 
Table I Agents and Corresponding Evidence from Studies Included in This Scoping Review (Listed in Descending Order by Number of Studies)

\begin{tabular}{|l|c|l|}
\hline Agent(s) & $\begin{array}{c}\text { Number } \\
\text { of Reports }\end{array}$ & $\begin{array}{l}\text { Number of Reports by } \\
\text { Subgroup }\end{array}$ \\
\hline Capsaicin & 9 & 2 RCT, I PCS, 3 CS, 3 CR \\
Morphine & 9 & 2 RCT, I PCS, 3 CS, 3 CR \\
Methadone & 6 & I RCT, 4 CS, I PCS \\
Cannabis & 3 & 2 PCS, I RCS \\
Gabapentin & 2 & 2 CS \\
Ketamine & 2 & I CR, I CS \\
Sevoflurane & 2 & 2 CR \\
ABHR (Ativan/Benadryl/Haldol/ & I & I CR \\
Reglan) & & \\
Acetylsalicylic acid (ASA) & I & I RCS \\
Amitriptyline & I & I RCS \\
Bupivacaine & I & I CS \\
Cellulose & I & I CS \\
Clonidine & I & I CS \\
Diamorphine hydrochloride & I & I RCS \\
Dexamethasone & I & I CR \\
Hydrocolloid & I & I CR \\
Methanol & I & I CS \\
Tetracaine & I CS \\
Turmeric or curcumin & I & I \\
\hline
\end{tabular}

Abbreviations: RCT, randomized controlled trial; PCS, prospective cohort study $\mathrm{RCS}$, retrospective comparative study; CS, case series; CR, case report.

\section{Discussion}

As described in the Introduction, there is a continuing unmet need for novel analgesic agents and routes of administration to improve patients' symptom control in hospice, palliative care, and cancer treatment.

Looking over the agents discussed in our present scoping review, it is possible that the benefit-to-risk ratio for topical analgesics in hospice, palliative or cancer care favors their wider use in patients whose concurrent medical conditions render them frail and prone to systemic toxicities from standard analgesic medications and routes of administration. We acknowledge that assays of blood levels of some topical agents may lack the sensitivity to detect them despite their apparent clinical effectiveness. ${ }^{50,51}$ It also appears evident that some agents, such as morphine, have greater analgesic effect when applied to areas of open skin compared with intact skin.

We undertook this scoping review to characterize the nature of the literature examining topical analgesics in these often challenging settings. We found that the small number of studies and the generally low quality of those studies limits the strength of evidence available upon which clinicians can rely.
This scoping review of off-label topical agents for pain control in patients in hospice, palliative or cancer care is distinct from NASEM's monograph on FDA-approved topical agents. This contrast exemplifies the tension between fundamentally different directions of evidencebased medicine. On one side are those who advocate for large trials designed to reduce bias associated with the investigators' or the participants' feelings and expectations, using statistical techniques and outcome measures deliberately applied to minimize the effect of outliers upon a study's conclusions. On the other side are those whose focus is on the personal (including individual biochemical, narrative and social) essence of the patient's experience particularly where "total pain" is concerned. The NASEM white paper deliberately excludes many trials as prone to bias and relies upon a selected subset of retrieved literature to shape clinical guidance for treating chronic noncancer pain. Unfortunately, some of the most pressing needs for pain therapies involve patients who are often unsuited to participate in rigorous, prolonged clinical trials to assess the efficacy of such agents. Given this imperative need, we undertook this study to identify potentially valuable therapies that may be overlooked or require years of slowly accruing clinical trials to enter the therapeutic armamentarium. These therapies, eg, topical agents, are not included as first- or second-line therapy in most treatment guidelines for cancer pain, eg, the WHO method despite clues in the published literature that they might be better introduced early in patients' care.

\section{Conclusion}

"You must go on. I can't go on. I'll go on." [The Unnamable (1953); Samuel Beckett, Nobel Laureate in Literature 1969]

Beckett's words express the dilemma faced by hospice, palliative and cancer care clinicians assessing the published evidence for guidance as to whether to try a topical agent for a patient with refractory severe pain. Our scoping review reveals a dearth of high-quality trials to provide such guidance. However, it is not acceptable to vacillate, waiting until the literature evolves sufficiently to permit accurate predictions of the effects and side effects of such therapies. Moreover, in recent years recognition that opioid-induced hyperalgesia and opioid use disorder have increased among the elderly ${ }^{52}$ has motivated a search for alternatives to systemic opioids - a longstanding pillar of palliative, hospice and cancer care. 
Common sense dictates that, just as it is hazardous to generalize from clinical observations of small groups of patients to the treatment of large populations, it is also hazardous to take results derived from pooled group responses and expect they will apply to each individual. For some time, palliative clinicians have practiced individualized medicine as population-based therapies frequently fail to control pain symptoms. Our scoping review of topical pain medications to consider as supplements to FDA-approved medications and routes when needed may offer clinicians ideas to aid in the management of patients with refractory severe pain in hospice, palliative and cancer care settings.

\section{Acknowledgments}

We thank Stacy Brady, RN and colleagues of the Providence (Rhode Island) Veterans Affairs Medical Center for their support (to BOT) during preparation of this manuscript.

\section{Disclosure}

The authors have no financial disclosures or conflicts of interest to report.

\section{References}

1. Singer AE, Meeker D, Teno JM, et al. Symptom trends in the last year of life from 1998 to 2010: a cohort study. Ann Intern Med. 2015;162 (3):175-183. doi:10.7326/M13-1609

2. van den Beuken-van Everdingen MH, Hochstenbach LM, Joosten EA, Tjan-Heijnen VC, Janssen DJ. Update on prevalence of pain in patients with cancer: systematic review and meta-analysis. $J$ Pain Symptom Manag. 2016;51(6):1070-1090.e9. doi:10.1016/j. jpainsymman.2015.12.340

3. Mitra R, Jones S. Adjuvant analgesics in cancer pain: a review. $A m$ $J$ Hosp Palliat Care. 2012;29(1):70-79. doi:10.1177/10499091 11413256

4. Kurita GP, Sjogren P, Klepstad P, Mercadante S. Interventional techniques for the management of cancer-related pain: clinical and critical aspects. Cancers. 2019;11(4):443. doi:10.3390/cancers 11040443

5. Munn Z, Peters MDJ, Stern C, et al. Systematic review or scoping review? Guidance for authors when choosing between a systematic or scoping review approach. BMC Med Res Methodol. 2018;18(1):143. doi:10.1186/s12874-018-0611-x

6. Winegarden JA, Carr DB, Bradshaw YS. Topical ketamine with other adjuvants: underutilized for refractory cancer pain? a case series and suggested revision of the World Health Organization stepladder for cancer pain. J Palliat Med. 2020;23(9):1167-1171. doi:10.1089/ jpm.2019.0618

7. The National Academies of Sciences, Engineering and Medicine. Compounded Topical Pain Creams, Review of Select Ingredients for Safety, Effectiveness, and Use. Consensus Study Report. Washington, DC: The National Academies Press; 2020.

8. Arksey H, O'Malley L. Scoping studies: towards a methodological framework. Int J Soc Res. 2005;8(1):19-32.

9. Peters MDJ, Godfrey CM, Khalil H, et al. Guidance for conducting systematic scoping reviews. Int J Evid Based Healthc. 2015;13 (3):141-146. doi:10.1097/XEB.0000000000000050
10. Jorge LL, Feres CC, Teles VE. Topical preparations for pain relief: efficacy and patient adherence. J Pain Res. 2010;4:11-24. doi:10.2147/JPR.S9492

11. Moher D, Liberati A, Tetzlaff J, Altman DG. Preferred reporting items for systematic reviews and meta-analyses: the PRISMA statement. PLoS Med. 2009;6(7):e1000097. doi:10.1371/journal. pmed.1000097

12. Hurgon A, Ferry I, Allot E, Dumas L. Topical morphine in treatment of painful malignant wounds: efficacy and safety for 7 cases. [Abstract]. Pharm World Sci. 2010;32(5):671.

13. Norman C, Maynard L. Buccal opioids for breakthrough pain in children with life-limiting conditions receiving end-of-life care. Int $J$ Palliat Nurs. 2019;25(10):472-479. doi:10.12968/ ijpn.2019.25.10.472

14. Allen Ma, MacDougall R, Murphy M, Robertson S. Atomized methadone suspension on the buccal mucosa at the end of life: an innovative delivery route. [AAHPM abstract P184]. J Pain Symptom Manage. 2016;52(6):E115-E116. doi:10.1016/j.jpainsymman.20 16.10.250

15. Allen MA, MacDougall R, Murphy M, Robertson S. Effectiveness of atomized methadone on the buccal mucosa in the last days of life: an innovative delivery route when patients can no longer swallow. J Palliat Care Med. 2016;6(2):1-5. doi:10.4172/2165-7386.1000250

16. Spaner D. Effectiveness of the buccal mucosa route for methadone administration at the end of life. $J$ Palliat Med. 2014;17 (11):1262-1265. doi:10.1089/jpm.2013.0522

17. Love R, Bourgeois K. Topical methadone: an alternative for pain control in end-of-life management. $J$ Palliat Med. 2014;17(2):128. doi:10.1089/jpm.2013.0517

18. Zeppetella G, Paul J, Ribeiro MD. Analgesic efficacy of morphine applied topically to painful ulcers. J Pain Symptom Manage. 2003;25 (6):555-558. doi:10.1016/S0885-3924(03)00146-5

19. Krajnik M, Zylicz Z, Finlay I, Łuczak J, van Sorge AA. Potential uses of topical opioids in palliative care-report of 6 cases. Pain. 1999;80(1):121-125. doi:10.1016/S0304-3959(98)00211-5

20. Krajnik M, Zylicz Z. Topical morphine for cutaneous cancer pain. J Palliat Med. 1997;11(4):325.

21. Ciałkowska-Rysz A, Dzierżanowski T. Topical morphine for treatment of cancer-related painful mucosal and cutaneous lesions: a double-blind, placebo-controlled cross-over clinical trial. Arch Med Sci. 2019;15(1):146. doi:10.5114/aoms.2018.72566

22. Miyazaki T, Satou S, Ohno T, Yoshida A, Nishimura K. Topical morphine gel for pain management in head and neck cancer patients. Auris Nasus Larynx. 2014;41(5):496-498. doi:10.1016/j. anl.2014.05.016

23. Discala SL, Basri D, Vartan CM, Silverman Ma. Use of topical morphine to relieve painful pressure ulcers. Consult Pharm. 2018;33(4):215-221. doi:10.4140/TCP.n.2018.215

24. Zeppetella G, Porzio G, Aielli F. Opioids applied topically to painful cutaneous malignant ulcers in a palliative care setting. J Opioid Manag. 2007;3(3):161-166. doi:10.5055/jom.2007.0054

25. Twillman RK, Long TD, Cathers TA, Mueller DW. Treatment of painful skin ulcers with topical opioids. J Pain Symptom Manage. 1999;17(4):288-292. doi:10.1016/S0885-3924(98)00140-7

26. Ramnarine S, Laird B, Williams LJ, Fallon MT. Investigating high-concentration $8 \%$ capsaicin patch in chronic cancer-treatment related peripheral neuropathic pain. J Clin Oncol. 2016;34(26):S208. doi:10.1200/jco.2016.34.26_suppl.208

27. Bhaskar A, England J, Lowe J. Management of neuropathic pain in cancer patients with $8 \%$ capsaicin patch- a preliminary case series. Eur J Pain Suppl. 2011;5(S1):84. doi:10.1016/S1754-3207(11) 70286-3

28. Thomas SE, Laycock H. The use of high dose topical capsaicin in the management of peripheral neuropathy: narrative review and local experience. $\mathrm{Br} \quad J \quad$ Pain. 2020;14(2):133-140. doi:10.1177/ 2049463720914332 
29. Borgeat A. Topical capsaicin in severe neuropathic pain. J Pain Palliat Care Pharmacother. 2012;26(2):190-192. doi:10.3109/ 15360288.2012.681842

30. Paice JA, Ferrans CE, Lashley FR, Shott S, Vizgirda V, Pitrak D. Topical capsaicin in the management of HIV-associated peripheral neuropathy. $J$ Pain Symptom Manage. 2000;19(1):45-52. doi:10.1016/S0885-3924(99)00139-6

31. Stavropoulou E, Zis P, Karra A, Isaia C, Vadalouka A. Capsaicin Patch $8 \%$ Use in Intractable Neuropathic Pain. World Institute of Pain Abstract Book; 2014. WIP-0589.

32. Wagner T, Roth-Daniek A, Sell A, England J, Kern KU. Capsaicin $8 \%$ patch for peripheral neuropathic pain: review of treatment best practice from 'real-world' clinical experience. Pain Manage. 2012;2 (3):239-250. doi:10.2217/pmt.12.13

33. Zis P, Bernali N, Argira E, Siafaka I, Vadalouka A. Effectiveness and impact of capsaicin $8 \%$ patch on quality of life in patients with lumbosacral pain: an open-label study. Pain Physician. 2016;19(7): E1049-E1053. doi:10.36076/ppj/2016.19.E1049

34. Horváth K, Boros M, Bagoly T, et al. Analgesic topical capsaicinoid therapy increases somatostatin-like immunoreactivity in the human plasma. Neuropeptides. 2014;48(6):371-378. doi:10.1016/j. npep.2014.10.001

35. Wilson M, Masterson E, Broglio K. Cannabis use among patients prescribed opioids in a palliative care clinic. [AAHPM abstract 875]. $J$ Pain Symptom Manage. 2019;57(2):522. doi:10.1016/j. jpainsymman.2018.12.325

36. Maida V, Corban J. Topical medical cannabis: a new treatment for wound pain-three cases of pyoderma gangrenosum. J Pain Symptom Manage. 2017;54(5):732-736. doi:10.1016/j. jpainsymman.2017.06.005

37. Highet BH, Lesser ER, Johnson PW, Kaur JS. Tetrahydrocannabinol and cannabidiol use in an outpatient palliative medicine population. Am J Hosp Palliat Med Care. 2020;37(8):589-593. doi:10.1177/ 1049909119900378

38. Vadaurri V. Topical treatment of neuropathic pain. Int J Pharm Compd. 2008;12(3):182.

39. Snyder S. Recent advances in extemporaneous compounding in unusual pain medications for hospice/terminally ill patients. Pain Digest. 1994;4:29-42.

40. Kopsky DJ, Liebregts R, Keppel Hesselink JM. Central neuropathic pain in a patient with multiple sclerosis treated successfully with topical amitriptyline. Case Rep Med. 2012;2012:471835. doi: $10.1155 / 2012 / 471835$
41. Schmelz M, Kress M. Topical acetylsalicylate attenuates capsaicin induced pain, flare and allodynia but not thermal hyperalgesia. Neurosci Lett. 1996;214(1):72-74. doi:10.1016/0304-3940(96) 12868-8

42. Whidden B. The use of dermafill cellulose wound-care dressing for management of pain in wounds in the hospice and palliative care setting. J Pain Symptom Manage. 2011;41(1):223-224. doi:10.1016/j. jpainsymman.2010.10.102

43. Kiedrowski M. Severe pain from shingles: a hydrocolloid dressing helps. BMJ Support Palliat Care. 2020. bmjspcare-2020-002447. doi:10.1136/bmjspcare-2020-002447

44. Slatkin NE, Rhiner M. Topical ketamine in the treatment of mucositis pain. Pain Med. 2003;4(3):298-303. doi:10.1046/j.15264637.2003.03032.x

45. Cortellini A, Verna L, Cannita K, et al. Topical menthol for treatment of chemotherapy-induced peripheral neuropathy. Indian J Palliat Care. 2017;23(3):350-352. doi:10.4103/IJPC.IJPC_23_17

46. Fernández-Ginés FD, Cortiñas-Sáenz M, de Aranda ANG, et al. Palliative analgesia with topical sevoflurane in cancer-related skin ulcers: a case report. Eur J Hosp Pharm. 2019;26(4):229-232. doi:10.1136/ejhpharm-2017-001421

47. Fernández-Ginés FD, Cortiñas-Sáenz M, Selva-Sevilla C, GerónimoPardo M. Sevoflurane topical analgesia for intractable pain with suicidal ideation. BMJ Support Palliat Care. 2020;bmjspcare-2019002023. doi:10.1136/bmjspcare-2019-002023

48. Shah N, Shah A, Lewis-Ramos V. "Ay doctor, deme algo porfavor'skin failure with Intractable pruritus. J Pain Symptom Manage. 2018;56(6):117-118. doi:10.1016/j.jpainsymman.2018.10.385

49. Kuttan R, Sudheeran PC, Josph CD. Turmeric and curcumin as topical agents in cancer therapy. Tumori. 1987;73(1):29-31. doi: $10.1177 / 030089168707300105$

50. Smith TJ, Ritter JK, Poklis JL, et al. ABH gel is not absorbed from the skin of normal volunteers. J Pain Symptom Manage. 2012;43 (5):961-966. PMID: 22560361. doi:10.1016/j.jpainsymman.2011.05.017

51. Paice JA, Von Roenn JH, Hudgins C, et al. Morphine bioavailability from a topical gel formulation in volunteers. J Pain Symptom Manage. 2008;35(3):314-320. doi:10.1016/j.jpainsymman.2007.04.016

52. Zullo AR, Danko KJ, Moyo P, et al. Prevention, diagnosis, and management of opioids, opioid misuse, and opioid use disorder in older adults. Rockville (MD): Agency for Healthcare Research and Quality (US); 2020. (Technical Brief, No. 37). Available from: https://www.ncbi.nlm. nih.gov/books/NBK564144/. Accessed September 9, 2021.
Journal of Pain Research

\section{Publish your work in this journal}

The Journal of Pain Research is an international, peer reviewed, open access, online journal that welcomes laboratory and clinical findings in the fields of pain research and the prevention and management of pain. Original research, reviews, symposium reports, hypothesis formation and commentaries are all considered for publication. The manuscript

Submit your manuscript here: https://www.dovepress.com/journal-of-pain-research-journa management system is completely online and includes a very quick and fair peer-review system, which is all easy to use. Visit http:// www.dovepress.com/testimonials.php to read real quotes from published authors. 